\title{
Almost periodic solutions of difference equations with discrete argument on metric space
}

\author{
Vasyl Slyusarchuk
}




\title{
ALMOST PERIODIC SOLUTIONS OF DIFFERENCE EQUATIONS WITH DISCRETE ARGUMENT ON METRIC SPACE
}

\author{
V. YU. SLYUSARCHUK
}

Received 04 January, 2014

\begin{abstract}
We obtain conditions for existence of almost periodic solutions of difference equations with discrete argument on metric space.

2010 Mathematics Subject Classification: 39A10; 47B39; 54J35

Keywords: nonlinear difference equations, almost periodic solutions, metric spaces
\end{abstract}

\section{INTRODUCTION}

Let $M$ be a metric space with metric $\rho_{M}$. Fix an arbitrary element $a \in M$. Denote by $\mathfrak{M}$ the metric space of sequences $\mathbf{x}=(\mathbf{x}(n))_{n \in \mathbb{Z}}$ for which

$$
\sup _{n \in \mathbb{Z}} \rho_{M}(\mathbf{x}(n), a)<\infty
$$

with metric

$$
\rho_{\mathfrak{M}}\left(\mathbf{x}_{1}, \mathbf{x}_{2}\right)=\sup _{n \in \mathbb{Z}} \rho_{M}\left(\mathbf{x}_{1}(n), \mathbf{x}_{2}(n)\right) .
$$

Define the shift operator $S_{m}: \mathfrak{M} \rightarrow \mathfrak{M}, m \in \mathbb{Z}$, by the formulae

$$
\left(S_{m} \mathbf{x}\right)(n)=\mathbf{x}(n+m), n \in \mathbb{Z} .
$$

Definition 1. The sequence $\mathbf{x} \in \mathfrak{M}$ is called almost periodic if the set $\overline{\left\{S_{m} \mathbf{x}: m \in \mathbb{Z}\right\}}$ is compact in $\mathfrak{M}$.

Denote by $\mathfrak{B}$ the metric space of sequences $\mathbf{x} \in \mathfrak{M}$ which are almost periodic.

Let $\mathcal{K}$ be the set of compact sets $K$ of the metric space $M$ and let $R(\mathbf{x})$ be the set $\{\mathbf{x}(n): n \in \mathbb{Z}\}$. Fix an arbitrary compact set $K \subset \mathcal{K}$. We denote by $\mathfrak{D}_{K}$ the set of all elements of $\mathbf{x} \in \mathfrak{M}$ for each of which $R(\mathbf{x}) \subset K$.

Definition 2. The operator $\mathbf{H}: \mathfrak{M} \rightarrow \mathfrak{M}$ is called almost periodic if for every set $K \in \mathcal{K}$ and a sequence $\left(m_{k}\right)_{k \geqslant 1}$ of whole numbers there exists a subsequence $\left(m_{k_{l}}\right)_{l \geqslant 1}$, which

$$
\lim _{l_{1} \rightarrow \infty, l_{2} \rightarrow \infty} \sup _{\mathbf{x} \in \mathfrak{D}_{K}} \rho_{\mathfrak{M}}\left(S_{m_{l_{1}}} \mathbf{H} S_{-m_{l_{1}}} \mathbf{x}, S_{m_{l_{2}}} \mathbf{H} S_{-m_{l_{2}}} \mathbf{x}\right)=0 .
$$


Note that the almost periodic operator $\mathbf{H}: \mathfrak{M} \rightarrow \mathfrak{M}$ can not be almost periodic by Bochner [2,3]. However, the almost periodic by Bochner operator $\mathbf{H}: \mathfrak{M} \rightarrow \mathfrak{M}$ be almost periodic by Definition 2.

Let $\Lambda$ be a bounded subset of the space $M$. Define the diameter diam $\Lambda$ of the set $\Lambda$ by the equality

$$
\operatorname{diam} \Lambda=\sup \left\{\rho_{M}(x, y): x, y \in \Lambda\right\} .
$$

Consider the almost periodic difference operator $\mathbf{F}: \mathfrak{M} \rightarrow \mathfrak{M}$ defined by the formulae

$$
(\mathbf{F x})(n)=F\left(n, \mathbf{x}(n), \mathbf{x}\left(n+m_{1}\right), \ldots, \mathbf{x}\left(n+m_{k}\right)\right), n \in \mathbb{Z},
$$

where $\mathbf{x} \in \mathfrak{M}, k \in \mathbb{N}, m_{1}, \ldots, m_{k} \in \mathbb{Z}$ and $F: \mathbb{Z} \times M^{k+1} \rightarrow M$ is operator such that

$$
\operatorname{diam} F\left(\mathbb{Z} \times M_{1} \times \ldots \times M_{k+1}\right)<+\infty
$$

for all bounded sets $M_{1}, \ldots, M_{k+1}$.

Consider the difference equation

$$
\mathbf{F x}=\mathbf{h},
$$

where $\mathbf{h} \in \mathfrak{B}$.

The aim of this work is to find conditions under which the bounded solutions of equation (1.1) are almost periodic.

In the study of equation (1.1) will use a functional defined on the set of solutions of an equation of the sets of values which are subsets of compact sets.

\section{FunCtional $\delta$}

Fix an arbitrary set $K \in \mathcal{K}$. Let $N(\mathbf{F}, K)$ be the set of all solutions of equation (1.1), each of which $R(\mathbf{x}) \subset \mathcal{K}$ and $\overline{R(\mathbf{x})} \neq K$. Suppose that $N(\mathbf{F}, K) \neq \varnothing$.

Fix an arbitrary element $\mathbf{x}^{*} \in N(\mathbf{F}, K)$. Let

$$
r\left(\mathbf{x}^{*}, K\right)=\sup \left\{\rho_{M}(x, y): x \in \overline{R\left(\mathbf{x}^{*}\right)}, y \in K\right\} .
$$

Due to the inequality $N(\mathbf{F}, K) \neq \varnothing$

$$
r\left(\mathbf{x}^{*}, K\right)>0 .
$$

Also fix the arbitrary number $\varepsilon \in\left[0, r\left(\mathbf{x}^{*}, K\right)\right]$. We denote by $\Omega\left(\mathbf{x}^{*}, K, \varepsilon\right)$ the set of all elements of $\mathbf{y} \in \mathfrak{M}$, each of which

$$
R(\mathbf{y}) \subset K
$$

and

$$
\rho_{\mathfrak{M}}\left(\mathbf{y}, \mathbf{x}^{*}\right) \geq \varepsilon .
$$

Consider the functional

$$
\delta\left(\mathbf{x}^{*}, K, \varepsilon\right)=\inf _{\mathbf{y} \in \Omega\left(\mathbf{x}^{*}, K, \varepsilon\right)} \rho_{\mathfrak{M}}\left(\mathbf{F y}, \mathbf{F} \mathbf{x}^{*}\right) .
$$


First analogous functionals have been proposed by the author in the papers $[7,8,10]$ for the study of nonlinear almost periodic equations

$$
\begin{aligned}
x(t+1) & =f(t, x(t)), \quad t \in \mathbb{R}, \\
\frac{d x(t)}{d t} & =f(t, x(t)), \quad t \in \mathbb{R},
\end{aligned}
$$

and

$$
f(t, x(t))=0, t \in \mathbb{R}
$$

with continuous operator $f: \mathbb{R} \times E \rightarrow E$. Here $E$ is a Banach space. Analogous functional for nonlinear difference equation

$$
x(n+1)=g(n, x(n)), n \in \mathbb{Z},
$$

used in [9].

\section{Main Result}

We give conditions for the existence of almost periodic solutions of equation (1.1), in contrast to the well-known theorem Amerio of almost periodic solutions of nonlinear differential equations (see $[1,4]$ ) do not use the H-class of equation (1.1). In the case of linear differential equations using $\mathrm{H}$-classes of these equations is essential $[5,6]$.

The main result of this paper reads as follows

Theorem 1. Let us suppose that $K \in \mathcal{K}, \mathbf{z} \in N(\mathbf{F}, K)$, diam $R(\mathbf{z}) \neq 0$ and

$$
\delta(\mathbf{z}, K, \varepsilon)>0
$$

for each $\varepsilon \in(0, r(\mathbf{z}, K))$. Then solution $\mathbf{z}$ of equation (1.1) is almost periodic.

Proof. Assume that the solution $\mathbf{z} \in N(\mathbf{F}, K)$ of the equation (1.1) is not element of the space $\mathfrak{B}$. Then there exists a sequence $\left(S_{m_{p}} \mathbf{z}\right)_{p \geq 1}$ such that each subsequence $\left(S_{k_{p}} \mathbf{z}\right)_{p \geq 1}$ is divergent. Consequently, for some numbers $p_{r} \in \mathbb{N}, q_{r} \in \mathbb{N}, r \geq 1$, and $\gamma \in(0, \operatorname{diam} R(\mathbf{z}))$

$$
\rho_{\mathfrak{M}}\left(S_{k_{p r}} \mathbf{z}, S_{k_{q r}} \mathbf{z}\right) \geq \gamma, \quad r \geq 1
$$

Because

and therefore

$$
\rho_{\mathfrak{M}}\left(\mathbf{z}, S_{-k_{p r}} S_{k_{q r}} \mathbf{z}\right) \geq \gamma, \quad r \geq 1
$$

$$
S_{-k_{p_{r}}} S_{k_{q_{r}}} \mathbf{z} \in \Omega(\mathbf{z}, K, \gamma), \quad r \geq 1
$$

Based on the inclusion of $\mathbf{h} \in \mathfrak{B}$, without loss of generality of the proof, we can assume that

$$
\lim _{r \rightarrow \infty} \rho_{\mathfrak{M}}\left(S_{-k_{p r}} \mathbf{h}, S_{-k_{q r}} \mathbf{h}\right)=0
$$


Note that diam $R(\mathbf{z})) \leq r(\mathbf{z}, K))$. Without loss of generality we can assume that the sequence $\left(S_{k_{p}} \mathbf{F} S_{-k_{p}} \mathbf{x}\right)_{p \geq 1}$ converges uniformly on $\mathfrak{D}_{K}$. Then

$$
\lim _{p, q \rightarrow \infty} \sup _{\mathbf{x} \in \mathfrak{D}_{K}} \rho_{\mathfrak{M}}\left(S_{k_{p}} \mathbf{F} S_{-k_{p}} \mathbf{x}, S_{k_{q}} \mathbf{F} S_{-k_{q}} \mathbf{x}\right)=0 .
$$

We show that

$$
\delta(\mathbf{z}, K, \gamma)=0 .
$$

It is obvious that by (2.1) and (3.4)

$$
\delta(\mathbf{z}, K, \gamma)=\inf _{\mathbf{y} \in \Omega(\mathbf{z}, K, \gamma)} \rho_{\mathfrak{M}}(\mathbf{F y}, \mathbf{F z}) \leq \rho_{\mathfrak{M}}\left(\mathbf{F} S_{-k_{p_{r}}} S_{k_{q_{r}}} \mathbf{z}, \mathbf{F z}\right), \quad r \geq 1
$$

Note that

$$
\begin{aligned}
& \rho_{\mathfrak{M}}\left(\mathbf{F} S_{-k_{p_{r}}} S_{k_{q_{r}}} \mathbf{z}, \mathbf{F} \mathbf{z}\right)= \\
& =\rho_{\mathfrak{M}}\left(S_{-k_{p_{r}}}\left(S_{k_{p_{r}}} \mathbf{F} S_{-k_{p_{r}}}\right) S_{k_{q_{r}}} \mathbf{z}, S_{-k_{q_{r}}}\left(S_{k_{q_{r}}} \mathbf{F} S_{-k_{q_{r}}}\right) S_{k_{q_{r}}} \mathbf{z}\right) \\
& \leq \rho_{\mathfrak{M}}\left(S_{-k_{p_{r}}}\left(S_{k_{p_{r}}} \mathbf{F} S_{-k_{p_{r}}}\right) S_{k_{q_{r}}} \mathbf{z}, S_{-k_{p_{r}}}\left(S_{k_{q_{r}}} \mathbf{F} S_{-k_{q_{r}}}\right) S_{k_{q_{r}}} \mathbf{z}\right) \\
& +\rho_{\mathfrak{M}}\left(S_{-k_{p_{r}}}\left(S_{k_{q_{r}}} \mathbf{F} S_{-k_{q_{r}}}\right) S_{k_{q_{r}}} \mathbf{z}, S_{-k_{q_{r}}}\left(S_{k_{q_{r}}} \mathbf{F} S_{-k_{q_{r}}}\right) S_{k_{q_{r}}} \mathbf{z}\right) \\
& =\rho_{\mathfrak{M}}\left(\left(S_{k_{p r}} \mathbf{F} S_{-k_{p_{r}}}\right) S_{k_{q_{r}}} \mathbf{z},\left(S_{k_{q_{r}}} \mathbf{F} S_{-k_{q_{r}}}\right) S_{k_{q_{r}}} \mathbf{z}\right)+\rho_{\mathfrak{M}}\left(S_{-k_{p_{r}}} S_{k_{q_{r}}} \mathbf{h}, S_{-k_{q_{r}}} S_{k_{q_{r}}} \mathbf{h}\right) \\
& \leq \sup _{\mathbf{x} \in \mathfrak{D}_{K}} \rho_{\mathfrak{M}}\left(S_{k_{p_{r}}} \mathbf{F} S_{-k_{p_{r}}} \mathbf{x}, S_{k_{q_{r}}} \mathbf{F} S_{-k_{q_{r}}} \mathbf{x}\right)+\rho_{\mathfrak{M}}\left(S_{-k_{p_{r}}} \mathbf{h}, S_{-k_{q_{r}}} \mathbf{h}\right), r \geq 1 .
\end{aligned}
$$

Therefore, based on (3.3), (3.4) and (3.6) the equality (3.5) is true.

This relation contradicts (3.1).

Thus, the assumption that the solution $\mathbf{z} \in N(\mathbf{F}, K)$ of the equation (1.1) is not element of the space $\mathfrak{B}$, is false.

So, the proof is complete.

\section{REFERENCES}

[1] L. Amerio, "Soluzioni quasiperiodiche, o limital, di sistemi differenziali non lineari quasiperiodici, o limitati," Ann. mat. pura ed appl., vol. 39, pp. 97-119, 1955.

[2] S. Bochner, "Beitrage zur theorie der fastperiodischen, i teil: Funktionen einer variablen," Math. Ann., vol. 96, pp. 119-147, 1927.

[3] S. Bochner, "Beitrage zur theorie der fastperiodischen, ii teil: Funktionen mehrerer variablen," Math. Ann., vol. 96, pp. 383-409, 1927.

[4] B. P. Demydovych, Lectures on the theory matematycheskoy Stability. Moskva: Nauka, 1967 (in Russian)

[5] J. Favard, "Sur les équations différentielles a coefficients presque périodiques," Acta Math., vol. 51, pp. 31-81, 1927.

[6] B. M. Levitan, Almost-periodic functions. Moskva: Gostechisdat, 1953 (in Russian).

[7] V. Y. Slyusarchuk, "Conditions for the existence of almost periodic solutions of nonlinear differential equations in banach space," Ukrain. Math. J., vol. 65, no. 2, pp. 307-312 (in Ukrainian), 2013.

[8] V. Y. Slyusarchuk, "Conditions of almost periodicity of bounded solutions of nonlinear difference equations with continuous argument," Nelinijni Kolyvannya, vol. 16, no. 1, pp. 118-124 (in Ukrainian), 2013. 
[9] V. Y. Slyusarchuk, "Conditions of almost periodicity of bounded solutions of nonlinear difference equations with discrete argument," Nelinijni Kolyvannya, vol. 16, no. 3, pp. 416-425 (in Ukrainian), 2013.

[10] V. Y. Slyusarchuk, "Criterion for the existence of almost periodic solutions of nonlinear equations, which does not use the $\mathscr{H}$-classes of these equations," Bukovinian Math. J., vol. 1, no. 1-2, pp. 136-138 (in Ukrainian), 2013.

\section{Author's address}

V. Yu. Slyusarchuk

National University of Water Management and Natural Resources Application, Department of Higher Mathematics, 11 Soborna St., 33000 Rivne, Ukraine

E-mail address: V.E.Slyusarchuk@gmail.com 